\title{
Circulating CD4+CD25+ Regulatory T Cells in the Pathobiology of Ulcerative Colitis and Concurrent Primary Sclerosing Cholangitis
}

\author{
Murat Kekilli • Bilge Tunc $\cdot$ Yavuz Beyazit • \\ Mevlut Kurt • Ibrahim Koral Onal • \\ Aysel Ulker • Ibrahim Celalettin Haznedaroglu
}

Received: 20 July 2012/ Accepted: 1 December 2012/Published online: 10 January 2013

(C) The Author(s) 2013. This article is published with open access at Springerlink.com

\begin{abstract}
Background Immunopathogenetic features of primary sclerosing cholangitis (PSC) in ulcerative colitis (UC) still remains unclear. Peripheral blood $\mathrm{CD} 4+\mathrm{CD} 25+$ regulatory $\mathrm{T}$ cells have a key role in the induction and maintenance of peripheral self-tolerance and inhibit several organ-specific autoimmune diseases. Therefore, $\mathrm{CD} 4+\mathrm{CD} 25+\mathrm{T}$ cells are believed to play an essential role in autoimmune diseases. The aim of the present study is to analyze the role of $\mathrm{CD} 4+\mathrm{CD} 25+\mathrm{T}$ cells in the pathogenesis of UC-associated PSC.

Methods This study evaluated the levels of CD4+CD25+ $\mathrm{T}$ cells in peripheral blood mononuclear cells (PBMC) of 27 UC patients with PSC and 20 UC patients as controls. $\mathrm{CD} 4+\mathrm{CD} 25+\mathrm{T}$ cells were isolated from PBMC with a direct immunofluorescence technique, using mice monoclonal antibodies namely FITC-labeled anti-CD4 and PElabeled anti-CD25. In each patient, CD4+CD25+ T cells percentage in PBMC were studied by flow cytometry, and then the number of $\mathrm{CD} 4+\mathrm{CD} 25+\mathrm{T}$ cells were calculated.

Results Twenty-seven UC patients with PSC and 20 UC patients without PSC as controls were enrolled in the present study. The percentage of CD4+CD25+ regulatory T cells among PBMC were significantly elevated in UC + PSC
\end{abstract}

M. Kekilli $(\varangle) \cdot$ B. Tunc $\cdot$ Y. Beyazit · M. Kurt ·

I. K. Onal · A. Ulker

Department of Gastroenterology, Turkiye Yuksek Ihtisas

Training and Research Hospital, Kızılay Sok. No: 2,

06100 Sihhiye, Ankara, Turkey

e-mail: drkekilli@gmail.com

I. C. Haznedaroglu

Department of Hematology, Hacettepe University,

Ankara, Turkey patients compared with UC patients without PSC ( $p=$ $0.04)$.

Conclusions $\mathrm{CD} 4+\mathrm{CD} 25+\mathrm{T}$ cells were found to be elevated in UC patients with PSC suggesting a partial role of activated $\mathrm{T}$ cell response in the disease pathophysiology. Our findings imply that $\mathrm{CD} 4+\mathrm{CD} 25+$ regulatory $\mathrm{T}$ cells may play a key role in the immunopathogenesis of UCassociated PSC and may affect the therapeutic management of these diseases.

Keywords Ulcerative colitis - Primary sclerosing cholangitis $\cdot \mathrm{CD} 4+\mathrm{CD} 25+\mathrm{T}$ cells $\cdot$ Pathophysiology

\section{Introduction}

Ulcerative colitis (UC) associated with primary sclerosing cholangitis (PSC) is usually clinically mild [1]. However, the genesis of this enigmatic association could exhibit in the early stages of the disease and sometimes requires a liver transplantation [2, 3]. UC and PSC are considered as parts of autoimmune syndromes [4]. Long-term complications of that association may develop neoplastic developments probably due to immune aberrations [5-8]. Nevertheless, the exact immune mechanism leading to the concurrent association of UC and PSC is unknown.

Peripheral blood CD4+CD25+ regulatory $\mathrm{T}$ cells have a central role in the immunopathology of numerous clinical states including inflammation, autoimmunity, and neoplasia [9-12]. These unique elements of the regulatory immune system may significantly locate pathobiology of cancer, diabetes, and heightened inflammation. The simultaneous occurrence of UC and PSC shall be investigated in the immune inflammatory perspectives of the diseases. 
With this respect, the present study was designed in order to evaluate whether the levels of CD4+CD25+ alter in UC patients with PSC. The clarification of the complex network of immune-inflammatory mediators operating in the gut of patients with UC and the association with hepatobiliary system could lead to the identification of new diagnostic and therapeutic targets that could, in turn, drive the development of effective therapeutic management. To the best of our knowledge, this study is the first to investigate the level of CD4+CD25+ in UC patients, and we conclude that present study is important because it facilitates additional research into the immunopathogenesis of UC-associated PSC.

\section{Patients and Methods}

\section{Patients}

Twenty-seven patients with UC and PSC and 20 matched patients with UC attending at the Gastroenterology Clinic of Turkiye Yuksek Ihtisas Training and Research Hospital between February 2008 and January 2011 were enrolled in the study. Patients with UC and PSC were included in the study if they were confirmed to have both PSC and UC by wellcharacterized clinical, endoscopic, radiologic, and pathologic features and had $>1$ colonoscopy with multiple biopsies of all colonic segments. The diagnosis of PSC was based on cholestatic biochemical profile or clinical signs of cholestasis, compatible liver histology, repeatedly anti-mitochondrial antibody (AMA) negativity by indirect immunofluorescence (IIFL), Western blot or ELISA and/or multifocal strictures, segmental dilatations and duct ectasia - of the intra hepatic and/or extra hepatic bile ducts on endoscopic retrograde cholangiopancreatography (ERCP) or magnetic resonance cholangiography (MRCP) (Fig. 1) after excluding secondary causes of sclerosing cholangitis. Patients with UC but without PSC were matched for age, gender, and disease duration.
Demographics and medical history of all patients, both cases and controls, including age, gender, family history of PSC and autoimmune diseases, history of pancreatitis, and presentation with jaundice disease duration, drug intake, duration of UC, and treatment profiles were noted for each subject. Complete blood count, erythrocyte sedimentation rate (ESR), and C-reactive protein (CRP) and biochemical tests including bilirubin, alanine aminotransferase (ALT), aspartate aminotransferase (AST), alkaline phosphatase (ALP), and albumin were determined for all patients. All of the patients were given comprehensive information before they consented to participate in the study. The study was conducted in accordance with the guidelines of the Helsinki Declaration.

The PSC Mayo risk score was calculated as per http://www.mayoclinic.org/grist/mayomodel3.html. Mayo risk score $(R)$ is scored as: low risk $(\leq 0)$, intermediate risk $(0-2)$, or high risk $(\geq 2)$.

\section{Serum Samples}

Blood samples were collected from a peripheral vein after an overnight fast without using any anticoagulant and were subjected to centrifugation with the speed of 3,000 rounds/ $\min$ for $10 \mathrm{~min}$ (Nuvefuge $\mathrm{CN} 180$ ), at $4{ }^{\circ} \mathrm{C}$ to obtain serum. All serum samples were stored at $-80{ }^{\circ} \mathrm{C}$ immediately after separation from peripheral blood prior to analysis.

\section{Flow Cytometric Analyses}

Peripheral blood mononuclear cells (PBMC) of patients were separated by density gradient centrifugation using Ficoll (Ficoll-Paque; GE Healthcare Life Sciences, Milan, Italy). CD4+CD25+ T cells were isolated from PBMC with a direct immunofluorescence technique using mice monoclonal antibodies (Becton-Dickinson, Sunnyvale, LA, USA), namely fluorescein isothiocyanate (FITC)-labeled
Fig. 1 ERCP (a) and MRCP (b) imaging of a PBS patient showing multifocal strictures and segmental dilatations in the hepatobiliary tract
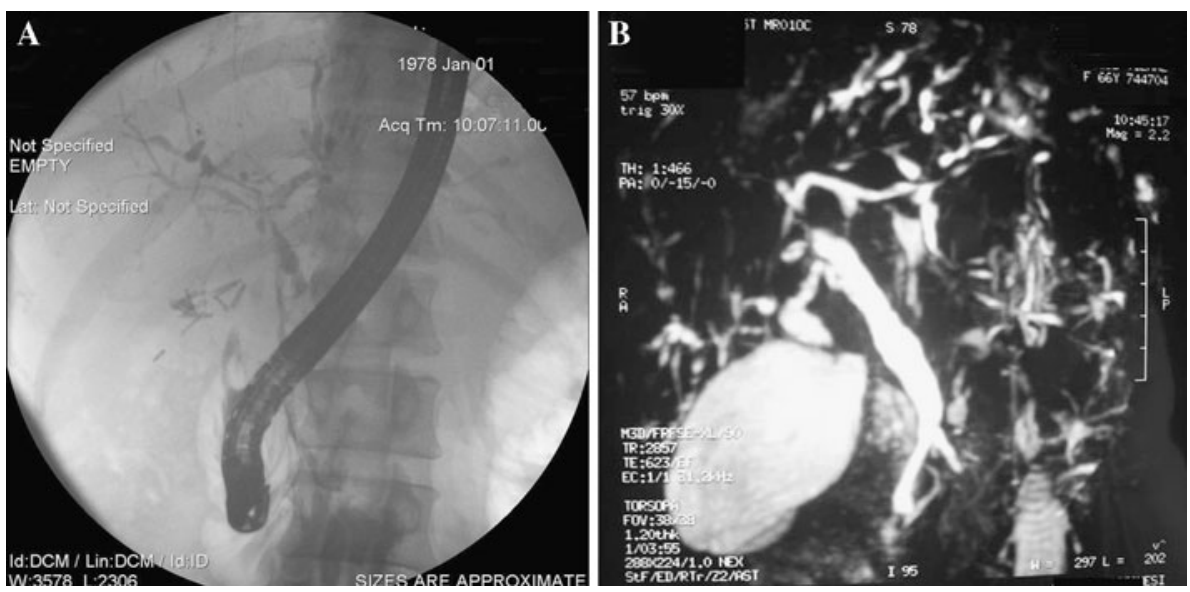
anti-CD4 and phycoerythrin (PE)-labeled anti-CD25. Phenotypes and proportions of each subpopulation were analyzed with aFACSCan (Becton-Dickinson, Sunnyvale, LA, USA) using Cell-Quest software (Becton-Dickinson, Sunnyvale, LA, USA).

\section{Statistical Analyses}

All statistical analyses were performed using SPSS statistical software package (SPSS Inc., Chicago, IL, USA). Comparison of different groups was done using the $\chi^{2}$ test or Fisher's exact test when appropriate. Mann-Whitney test was used for comparison of medians. Data found to be nonnormally distributed were analyzed using the nonparametric Mann-Whitney $U$ test (for two groups). The sensitivity and specificity of CD4+CD25+ T cells number for the detection of PSC in UC patients were calculated under various cut-off ranges, and the receiver operating characteristic (ROC) curves were drawn. A $p$ value of $<0.05$ was deemed statistically significant.

\section{Results}

Twenty-seven UC patients with PSC and 20 UC patients without PSC as controls were enrolled in the present study. There were 17 males and ten females (mean age $39.7 \pm 13.4$ years) in the UC + PSC group and 15 males and five females (mean age $36.1 \pm 12.8$ years) in the control group. The demographic characteristics of patients and control subjects were summarized in Table 1. There were no statistically significant differences between age, gender, and clinical characteristics of the study participants.

All of the patients with UC had been taking oral mesalamine. The patients with UC and PSC additionally had been taking oral ursodeoxycholic acid. None of the

Table 1 Demographic features of the study population

\begin{tabular}{llll}
\hline & $\mathrm{UC}(n=20)$ & $\begin{array}{l}\mathrm{UC}+\mathrm{PSC} \\
(n=27)\end{array}$ & $p$ \\
\hline Age (years) & $36.1 \pm 12.8$ & $39.7 \pm 13.4$ & $\mathrm{NS}$ \\
Gender (m/f) & $15 / 5$ & $17 / 10$ & $\mathrm{NS}$ \\
Smoking (+/-) & $5 / 15$ & $9 / 18$ & $\mathrm{NS}$ \\
Disease duration & $129.3 \pm 19.6$ & $122.25 \pm 17.21$ & $\mathrm{NS}$ \\
$\quad$ (months) & & & \\
Disease localization & & $7(26.0)$ & \\
$\quad$ Distal colitis, $n(\%)$ & $7(35)$ & $5(18.5)$ & \\
$\quad$ Left, $n(\%)$ & $10(50)$ & $15(55.5)$ & \\
Pancolitis, $n(\%)$ & $3(15)$ & & \\
\hline
\end{tabular}

NS not significant
Table 2 Clinical characteristics of the ulcerative colitis and concomitant primary sclerosing cholangitis patients

\begin{tabular}{lrr}
\hline & Number of patients & $\%$ \\
\hline Pruritus & 13 & 48.1 \\
Abdominal pain & 9 & 33.3 \\
Fatigue & 9 & 33.3 \\
Fever & 6 & 22.2 \\
Diarrhea & 5 & 18.5 \\
Surgery $^{\mathrm{a}}$ & 3 & 11.1 \\
Cholelithiasis $_{\text {Bacterial cholangitis }}{ }^{\mathrm{b}}$ & 5 & 18.5 \\
Dominant stricture $_{\text {Cirrhosis }}$ & 6 & 22.2 \\
& 2 & 7.4 \\
\hline
\end{tabular}

${ }^{\text {a }}$ Liver transplantation or biliary surgery

b Developed after the definitive diagnosis of PSC and accepted as complication

patients had a history or coexistence of malignant disease including cholangiocarcinoma and/or colon cancer. Various clinical characteristics of the patients with UC and concomitant PSC are listed in Table 2.

The Mayo risk score was calculated in 27 UC patients with PSC: 20 patients found to have a low-risk score, six had intermediate, and one had a high-risk score. No significant difference was observed in the mean number of CD4+CD25 + T cells between patients with UC and PSC in respect to Mayo risk scores $(p=0.283)$.

The Presence of CD4+CD25+ T Cells in UC Patients With or Without PSC

In each patient, $\mathrm{CD} 4+\mathrm{CD} 25+\mathrm{T}$ cells percentage in PBMC were studied by flow cytometry, and then the number of CD4+CD25+ T cells were calculated (Fig. 2).

The percentage of $\mathrm{CD} 4+\mathrm{CD} 25+$ regulatory $\mathrm{T}$ cells among PBMC in UC patients with PSC was $15.10 \pm 8.13$ and $10.58 \pm 6.33$ in UC patients without PSC $(p=0.04)$ (Fig. 3).

ROC curve analysis suggested that the optimum CD4+CD25+ T cells cut-off point for PSC in UC patients was 1.59 , with a sensitivity, specificity, positive predictive value, and negative predictive value of $74.1,55.0,69.0$, and $61.1 \%$ respectively [AUC: $0.66 \quad(95 \%$ CI 0.504-0.816), $p=0.04$ ] (Fig. 4). Overall accuracy of $\mathrm{CD} 4+\mathrm{CD} 25+\mathrm{T}$ cells in determination of PSC in UC patients was $65.0 \%$.

\section{Discussion}

In this study, we observed that the presence of CD4+CD25+ regulatory $\mathrm{T}$ cells among PBMC was significantly higher in 



Fig. 2 a Histogram indicating the cellular distribution of the increased $\mathrm{CD} 4+\mathrm{CD} 25+\mathrm{T}$ cells in the peripheral blood mononuclear cells (PBMC) derived from Ficoll density gradient centrifugation. The right upper quadrant of the diagram represents positively stained CD4+CD25+ T cells. b Histogram depicting the cellular distribution of PBMC that is negative for the population of CD4+CD25+ T cells

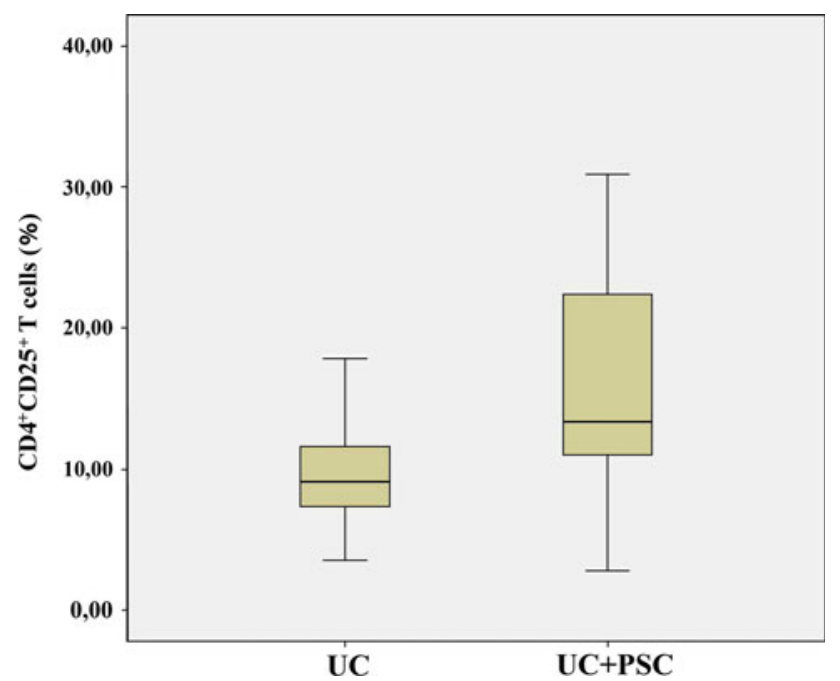

Fig. 3 Box plot presentation of CD4+CD25+ T cells among UC and $\mathrm{UC}+$ PBS patients

patients with UC and PSC in comparison to the patients with UC alone ( $p=0.04$ ). This novel finding cast attention on the immune mechanisms affecting the unique association of the two disease entities (i.e., UC and PSC). Inflammation, autoimmunity, and neoplasia represent the common points of cross-talk between CD4+CD25+ regulatory $\mathrm{T}$ cells among PBMC, UC, and PSC [9-12]. Those pathogenetic events also determine the clinical outcome of the patients with UC and/or PSC [1, 7, 8, 13-22]. The immunosuppressive properties of regulatory $\mathrm{T}$ cells have emerged as an attractive tool for the development of immunotherapies in various disease contexts, e.g., to treat transplantationinduced immune reactions [23]. Therefore, our findings about CD4+CD25+ regulatory T cells, UC, and PSC may also affect the management of those diseases based on future investigations in the battle field.

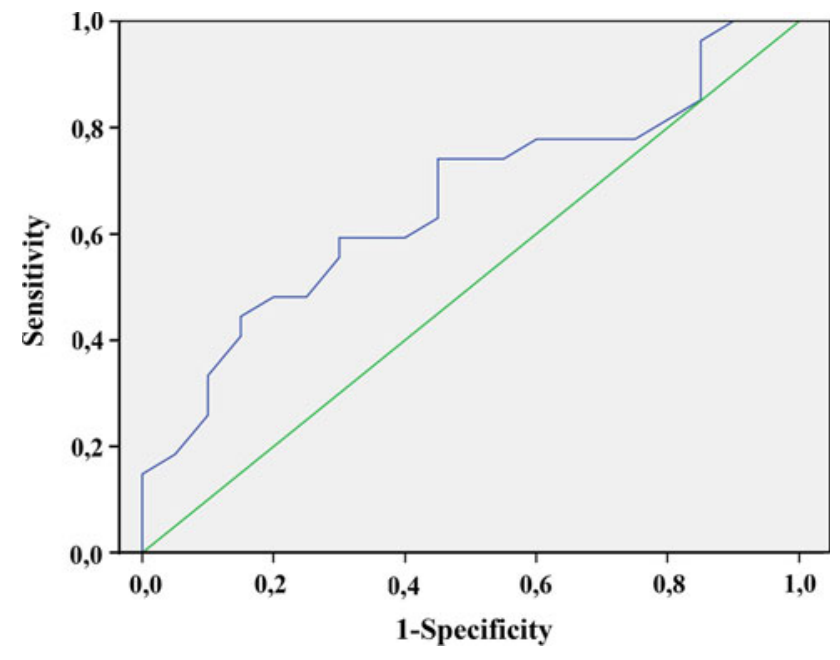

Fig. 4 Receiver operating characteristic (ROC) curve showing the sensitivity and specificity of CD4+CD25 $+\mathrm{T}$ cells for PBS in UC patients

Regulatory $\mathrm{T}$ cells are increased in context of malignancies and their expansion can be correlated with higher disease burden and decreased survival [24]. Long-term complications of the association UC and PSC may develop gastrointestinal neoplastic developments, such as colonic cancer probably due to immune aberrations [5-8]. In this study, we found that $\mathrm{CD} 4+\mathrm{CD} 25+$ regulatory $\mathrm{T}$ cells among PBMC was significantly higher in patients with UC and PSC in comparison to the patients with UC alone. However, neoplastic GI disease was not present in our case series. Our patients were receiving immunosuppression to prevent disease recurrence, avoiding at the same time the occurrence of "de novo" neoplasms as suggested in the literature [13]. Therefore the association between enhanced peripheral blood $\mathrm{CD} 4+\mathrm{CD} 25+$ regulatory $\mathrm{T}$ cells and neoplastic development in UC and PSC do not seem to be clear, particularly in patients under chronic treatment with immunosuppressive agents.

In vitro studies disclosed that following co-culture with autologous CD4+CD25+ responder $\mathrm{T}$ cells, human monocytes and monocyte-derived macrophages become activated but also significantly more prone to apoptosis than monocytes/macrophages cultured alone [9]. Pathological features of UC and PSC include somehow descriptive cellular alterations [15]. UC patients with PSC show a propensity for more extensive, but less active, disease but are otherwise characterized by similar pathologic findings compared with UC patients without PSC. Rectal sparing and patchy disease activity is not characteristic of UC patients with PSC [15]. The definitive role of monocyte-macrophages system in UC and/or PSC and their association between $\mathrm{CD} 4+\mathrm{CD} 25+$ regulatory $\mathrm{T}$ cells remain to be elucidated. 
Novel monoclonal antibodies affecting TNF signaling system and immunosuppressive drugs may alter the clinical course of inflammatory diseases [25-27]. None of our patients were receiving adalimumab, infliximab, or etanercept. Future investigations may also reveal whether the expansion of CD4+CD25+ responder $\mathrm{T}$ cells in patients with UC and PSC in comparison to the patients with UC alone could represent this subpopulation subject to novel immunomodulating therapies.

Regulatory $\mathrm{T}$ cell phenotypes suggest an intriguing role for immune regulatory mechanisms in numerous diseases in different ethnic groups [28]. The natural course of inflammatory bowel diseases also differs among different ethnic groups in distinct clinical settings [22]. In this study, Turkish patients with UC and PSC exhibited CD4+CD25+ regulatory $\mathrm{T}$ cells among PBMC. Similar investigations should be performed worldwide to detect unique immunological cellular alterations of the patients with inflammatory bowel diseases and their associations with clinical phenotypes.

In conclusion, we detected expansion of CD4+CD25+ regulatory $\mathrm{T}$ cells in the peripheral blood of patients with UC associated with PSC via flow cytometric analyses. Further experimental and clinical studies may shed further light on the enigmatic association of UC and PSC in the immune context of the diseases and may open new avenues for the better understanding and management of those difficult-to-treat clinical inflammatory diseases.

\section{Conflict of interest None.}

Open Access This article is distributed under the terms of the Creative Commons Attribution Noncommercial License which permits any noncommercial use, distribution, and reproduction in any medium, provided the original author(s) and the source are credited.

\section{References}

1. Marelli L, Xirouchakis E, Kalambokis G, et al. Does the severity of primary sclerosing cholangitis influence the clinical course of associated ulcerative colitis? Gut. 2011;60:1224-1228.

2. Navaneethan U, Venkatesh PG, Lashner BA, et al. The impact of ulcerative colitis on the long-term outcome of patients with primary sclerosing cholangitis. Aliment Pharmacol Ther. 2012. doi:10.1111/j.1365-2036.2012.05063.x.

3. Navaneethan U, Venkatesh PG, Mukewar S, et al. Progressive primary sclerosing cholangitis requiring liver transplantation is associated with reduced need for colectomy in patients with ulcerative colitis. Clin Gastroenterol Hepatol. 2012;10:540-546.

4. Farkas K, Nagy F, Kovacs L, et al. Ulcerative colitis and primary sclerosing cholangitis as part of autoimmune polyglandular syndrome type III. Inflamm Bowel Dis. 2010;16:10-11.

5. Eaton JE, Silveira MG, Pardi DS, et al. High-dose ursodeoxycholic acid is associated with the development of colorectal neoplasia in patients with ulcerative colitis and primary sclerosing cholangitis. Am J Gastroenterol. 2011;106:1638-1645.
6. Fukuhara T, Egashira A, Imamura M, et al. Proctocolectomy for colon cancer associated with ulcerative colitis a few months after living donor liver transplantation for primary sclerosing cholangitis: report of a case. Surg Today. 2009;39:59-63.

7. Lindstrom L, Lapidus A, Ost A, et al. Increased risk of colorectal cancer and dysplasia in patients with Crohn's colitis and primary sclerosing cholangitis. Dis Colon Rectum. 2011;54:1392-1397.

8. Navaneethan U, Venkatesh PG, Lashner BA, et al. Temporal trends in colon neoplasms in patients with primary sclerosing cholangitis and ulcerative colitis. J Crohns Colitis. 2012;6:845-851.

9. Jagger AL, Evans HG, Walter GJ, et al. FAS/FAS-L dependent killing of activated human monocytes and macrophages by $\mathrm{CD} 4+\mathrm{CD} 25-$ responder $\mathrm{T}$ cells, but not $\mathrm{CD} 4+\mathrm{CD} 25+$ regulatory T cells. $J$ Autoimmun. 2012;38:29-38.

10. Luopajarvi K, Nieminen JK, Ilonen $\mathrm{J}$, et al. Expansion of $\mathrm{CD} 4+\mathrm{CD} 25+\mathrm{FOXP} 3+$ regulatory $\mathrm{T}$ cells in infants of mothers with type 1 diabetes. Pediatr Diabetes. 2012;13:400-407.

11. Tran GT, Hodgkinson SJ, Carter NM, et al. Interleukin-5 (IL-5) promotes induction of antigen specific $\mathrm{CD} 4+\mathrm{CD} 25+\mathrm{T}$ regulatory cells that suppress autoimmunity. Blood. 2012;119:4441-4450.

12. Yamamoto $\mathrm{T}$, Yanagimoto $\mathrm{H}$, Satoi $\mathrm{S}$, et al. Circulating $\mathrm{CD} 4+\mathrm{CD} 25+$ regulatory $\mathrm{T}$ cells in patients with pancreatic cancer. Pancreas. 2012;41:409-415.

13. Bosso MC, Marchesa PE, Ricchiuti A, et al. Proctocolectomy for ulcerative colitis after liver transplantation for primary sclerosing cholangitis. Transplant Proc. 2009;41:1390-1392.

14. Itou M, Mitsuyama K, Kawaguchi T, et al. Leukocytapheresis therapy improved cholestasis in a patient suffering from primary sclerosing cholangitis with ulcerative colitis. Case Rep Gastroenterol. 2009;3:77-83.

15. Joo M, Abreu-e-Lima P, Farraye F, et al. Pathologic features of ulcerative colitis in patients with primary sclerosing cholangitis: a case-control study. Am J Surg Pathol. 2009;33:854-862.

16. Katsanos KH, Saougos V, Kosmidou M, et al. Sjögren's syndrome in a patient with ulcerative colitis and primary sclerosing cholangitis: case report and review of the literature. J Crohns Colitis. 2009;3:200-203.

17. Kawaguchi T, Kaji R, Horiuchi H, et al. Development of intrahepatic cholangiocarcinoma after a 14-year follow-up of a patient with primary sclerosing cholangitis and ulcerative colitis. Hepatol Res. 2011;41:1253-1259.

18. Kevans D, Greene J, Galvin L, et al. Mesalazine-induced bronchiolitis obliterans organizing pneumonia (BOOP) in a patient with ulcerative colitis and primary sclerosing cholangitis. Inflamm Bowel Dis. 2011;17:E137-E138.

19. Lepisto A, Kivisto S, Kivisaari L, et al. Primary sclerosing cholangitis: outcome of patients undergoing restorative proctocolecetomy for ulcerative colitis. Int J Colorectal Dis. 2009;24: $1169-1174$

20. Van Hauwaert V, Meers S, Verhoef G, et al. Rectal non-Hodgkin's lymphoma in an infliximab treated patient with ulcerative colitis and primary sclerosing cholangitis. J Crohns Colitis. 2010; 4:683-686.

21. Wasmuth $\mathrm{HH}$, Trano G, Endreseth BH, et al. Primary sclerosing cholangitis and extraintestinal manifestations in patients with ulcerative colitis and ileal pouch-anal anastomosis. $J$ Gastrointest Surg. 2001;14:1099-1104.

22. Ye BD, Yang SK, Boo SJ, et al. Clinical characteristics of ulcerative colitis associated with primary sclerosing cholangitis in Korea. Inflamm Bowel Dis. 2011;17:1901-1906.

23. Berglund $\mathrm{D}$, Korsgren $\mathrm{O}$, Lorant $\mathrm{T}$, et al. Isolation, expansion and functional assessment of $\mathrm{CD} 4+\mathrm{CD} 25+\mathrm{FoxP} 3+$ regulatory $\mathrm{T}$ cells and $\operatorname{Tr} 1$ cells from uremic patients awaiting kidney transplantation. Transpl Immunol. 2012;26:27-33.

24. Beyer M, Schumak B, Weihrauch MR, et al. In vivo expansion of naive CD4+CD25 (high) FOXP3+ regulatory T cells in patients 
with colorectal carcinoma after IL-2 administration. PLoS One. 2012;7:e30422.

25. Calleja S, Cordero-Coma M, Rodriguez E, et al. Adalimumab specifically induces CD3 (+) CD4 (+) CD25 (high) Foxp3 (+) CD127 (-) T-regulatory cells and decreases vascular endothelial growth factor plasma levels in refractory immuno-mediated uveitis: a non-randomized pilot intervention study. Eye (Lond). 2012;26:468-477.

26. Richetta AG, Mattozzi C, Salvi M, et al. Down regulation of circulating CD4 (+) CD25 (bright) Foxp3 (+) T cells by cyclosporine therapy and correlation with clinical response in psoriasis patients: report of three cases. Int J Dermatol. 2012;. doi:10.1111/j.13654632.2011.05411.x.

27. Wang GY, Yang Y, Li H, et al. Rapamycin combined with donor immature dendritic cells promotes liver allograft survival in association with CD4 (+) CD25 (+) Foxp3 (+) regulatory T cell expansion. Hepatol Res. 2012;42:192-202.

28. Sanou GS, Tiendrebeogo RW, Ouedraogo AL, et al. Hematological parameters, natural regulatory $\mathrm{CD} 4+\mathrm{CD} 25+\mathrm{FOXP} 3+\mathrm{T}$ cells and gamma delta $\mathrm{T}$ cells among two sympatric ethnic groups having different susceptibility to malaria in Burkina Faso. BMC Res Notes. 2012;5:76. 